\title{
Development and ordering of mounds during metal(100) homoepitaxy
}

\author{
K. J. Caspersen, A. R. Layson, C. R. Stoldt, V. Fournee, P. A. Thiel, and J. W. Evans \\ Departments of Chemistry and Mathematics, and Ames Laboratory, Iowa State University, Ames, Iowa 50011
}

(Received 11 February 2002; published 13 May 2002)

\begin{abstract}
Scanning-tunneling microscopy studies combined with atomistic modeling for $\mathrm{Ag} / \mathrm{Ag}(100)$ homoepitaxy reveal complex growth behavior at $300 \mathrm{~K}$ : initial smooth growth up to $\sim 25 \mathrm{ML}$, where three-dimensional (3D) mounds develop from 2D islands; then an extended regime of mound steepening for $\sim 1000$ ML producing unexpected rough growth; and finally an asymptotic regime with cooperative mound ordering and coalescence dynamics quite distinct from that in systems with up-down symmetry. The steepening regime is compressed upon lowering temperature, so while initial growth is rougher, asymptotic growth is actually smoother.
\end{abstract}

DOI: 10.1103/PhysRevB.65.193407

PACS number(s): 68.55.-a, 81.15.Aa, 68.35.Fx

The evolution of complex patterns in physical systems occurs in a variety of phase separation and domainboundary-driven coarsening processes. Examples can be found in alloy systems, complex fluids, and surface adlayers. ${ }^{1}$ There is considerable experimental and theoretical interest in analyzing these patterns, often focussing on longtime asymptotic behavior. The latter is of particular interest as it typically falls into one of a few "universality classes" determined by the basic characteristics of the process (symmetry, domain degeneracy, dimension) rather than by the finer details. ${ }^{1}$ Another example receiving much attention recently is formation of 3D mounds (multilayer stacks of 2D islands) during epitaxial growth, ${ }^{2-9}$ a phenomenon of significance for various thin film deposition technologies. An extra complication here is a subtle interplay between the observed lateral coarsening of the array of mounds, and the kinetic roughening of growing film. ${ }^{4-12}$ In fact, many aspects of the morphological "landscapes" developing in these growing films have yet to be carefully examined or fully understood. Thus, a basic goal is to develop atomistic models, which can predict quantitatively these morphologies.

Mound formation during epitaxial growth is often associated with step-edge (SE) barriers to downward transport, which promote reflection of diffusing atoms from descending steps and incorporation at ascending steps. This produces a destabilizing lateral mass current $J_{\text {up }}$ in the uphill direction. ${ }^{13}$ Mound slopes increase initially, but may stabilize if there is sufficient buildup of a downhill current $J_{\text {down }}$ (e.g., due to "downward funneling" of depositing atoms at step edges) counterbalancing $J_{\text {up }} \cdot{ }^{14}$ An extreme regime of mound formation is often found in metal(111) homoepitaxial systems due to large SE barriers. These produce the near-Poisson film height distributions for $\mathrm{Ag} / \mathrm{Ag}(111)$ (Refs. 3 and 4) and $\mathrm{Pt} / \mathrm{Pt}(111){ }^{5}$ with persistent mound steepening, as well as a lack of coarsening for $\mathrm{Pt} / \mathrm{Pt}(111) .^{5}$ Contrasting behavior is seen in metal(100) systems such as $\mathrm{Cu} / \mathrm{Cu}(100)$ (Ref. 6) and $\mathrm{Fe} / \mathrm{Fe}(100){ }^{7}$ where slope selection is rapid, tying mound coarsening to film roughening. Metal(100) systems are generally believed to have low SE barriers, prompting view that $\mathrm{Ag} / \mathrm{Ag}(100)$ growth is smooth, contrasting $\mathrm{Ag} / \mathrm{Ag}(111) .{ }^{15} \mathrm{We}$ shall show, however, that $\mathrm{Ag} / \mathrm{Ag}(100)$ growth is more complex than any of the above scenarios.

From a theoretical perspective, there is intense interest in characterizing asymptotic roughening and mound coarsening mechanisms. ${ }^{10-12}$ Simple continuum models with up-down symmetry have been applied to compare behavior in (111) and (100) systems, noting subtle topological effects in the latter. But do these reflect behavior in real systems which lack up-down symmetry? ${ }^{7,14}$ One should also scrutinize comparisons made with experiment, where data is often available only for a single temperature $(T)$ and limited range of film thickness.

In this paper, we provide a detailed and comprehensive picture of kinetic roughening and mound evolution for $\mathrm{Ag} / \mathrm{Ag}(100)$ film growth between 190 and $300 \mathrm{~K}$. This is achieved by integrating high-resolution low-energy electron diffraction (LEED) studies (up to $\sim 10$ monolayers or ML), variable-temperature scanning tunneling microscopy (VTSTM) studies (up to $\sim 100 \mathrm{ML}$ ), and kinetic Monte Carlo (KMC) simulations of a realistic atomistic growth model (up to $\sim 2000 \mathrm{ML}$ ). Modeling recovers all observed features of $25 \mathrm{ML}$ films deposited from 175 to $300 \mathrm{~K},{ }^{8,9}$ and is used to predict evolution of much thicker films. Furthermore, it elucidates key aspects of atomistic processes underlying growth (e.g., nonuniform SE barriers, inhibited kink rounding below $230 \mathrm{~K}$ ). As expected, initial growth is fairly smooth at $300 \mathrm{~K}$, and rougher at $\sim 200 \mathrm{~K}$, where small SE barriers inhibit smoothing. $4,8,9$ Contrary to common belief, we find that exactly the opposite is true for thick films. This is due to a regime of mound steepening and rapid roughening which extends for $\sim 1000 \mathrm{ML}$ at $300 \mathrm{~K}$, but which is much compressed at $\sim 200 \mathrm{~K}$. Our modeling also reveals subtle aspects of morphological evolution in the subsequent asymptotic slope-selection regime including complex mound ordering, annihilation, and coalescence dynamics, which reflects strong up-down symmetry breaking and which is very different from behavior observed in abovementioned continuum formulations of mounding.

Experiments were performed in a UHV chamber with base pressure $<10^{-10}$ Torr. Films were produced by evaporative deposition of $\mathrm{Ag}$ onto the $\mathrm{Ag}(100)$ single-crystal surface between 190 and $300 \mathrm{~K}$, the temperature regime of mound formation, ${ }^{8}$ with a flux of $F \approx 0.02 \mathrm{ML} / \mathrm{s}$. Nanostructure evolution was monitored with an Omicron VTSTM. Specifically, we determine the film roughness $W$ (in units of the interlayer spacing $b=2.04 \AA$ ) and the height-difference correlation function $H(r)$ (mean-square surface height difference versus lateral separation $r$ ). Oscillations in the latter reflect a partially ordered array of mounds, the first minimum 


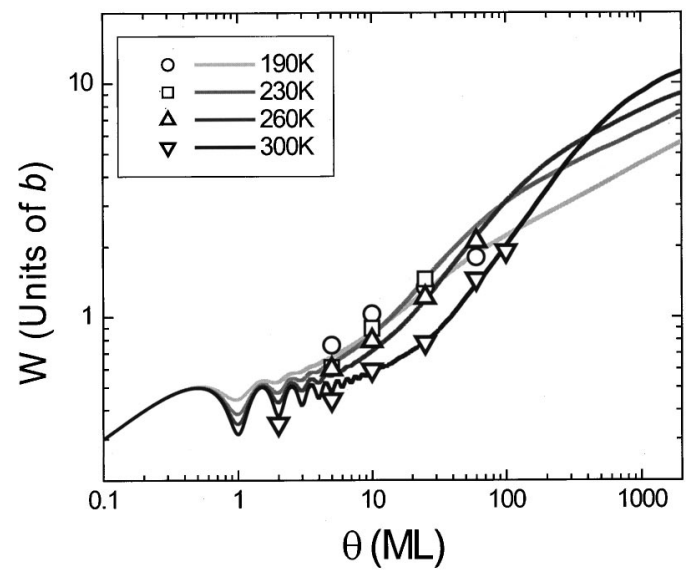

FIG. 1 . $W$ vs coverage, $\theta$, for growth at different $T$ (shown). Experimental data (symbols) and model predictions (curves from bottom to top—on right—with increasing $T$ ).

roughly corresponding to the mean mound "diameter" $L_{D}$. We also directly determine the mound density $N_{m}$ and thus the mean mound separation $L_{S}=1 / \sqrt{N_{m}}$. For the initial stage of growth, VTSTM statistics for $H(r)$ are poorer, so we instead extract lateral correlation lengths $L_{C} \propto 1 / d^{*}$, where $d^{*}$ is the inverse ring diameter of LEED profiles obtained during deposition in a separate UHV chamber. We have previously characterized the morphology of $25 \mathrm{ML}$ films versus $T,{ }^{8}$ but all the results reported here for kinetic roughening and mound coarsening are new.

First, we describe our VTSTM results for kinetic roughening, the interpretation of which is facilitated by comparison with results from KMC simulations of our atomistic model described below. Experimental data (symbols) and simulation results (curves) for $W$ versus coverage $\theta$ are shown in Fig. 1 for various $T$. At $300 \mathrm{~K}$, one finds distinct regimes of initial "smooth" growth up to $\sim 25$ ML (consistent with Ref. 4), rapid "preasymptotic" roughening up to $\sim 1500$ ML (during which mounds steepen), and subsequent "asymptotic" slope selection (as checked in simulations) with slower roughening. The three regimes are compressed upon reducing $T$, so that asymptotic behavior is already achieved by $\sim 100 \mathrm{ML}$ at $190 \mathrm{~K}$. The unexpected feature of very rough (asymptotic) growth at $300 \mathrm{~K}$, compared with smoother growth at $\sim 200 \mathrm{~K}$, is explained below. Roughening behavior can be described in terms of an effective exponent $\beta_{\text {eff }}=d(\ln W) / d(\ln \theta)$, which varies strongly with $\theta$ for higher $T$, but less so at $190 \mathrm{~K}$. From simulations, $\beta_{\text {eff }}$ has low values in the initial regime (e.g., $\beta_{\text {eff }} \approx 0.2$ at $300 \mathrm{~K}$ ), high values in the preasymptotic regime (which peak at $\beta_{\text {eff }} \approx 0.8$ at $300 \mathrm{~K}, 0.75$ at $260 \mathrm{~K}, 0.65$ at $230 \mathrm{~K}$, and 0.45 at $190 \mathrm{~K}$ ), and lower "asymptotic" values of $\beta_{\text {eff }} \approx 0.3$ at $190 \mathrm{~K}$, and $\sim 0.25$ for higher $T$. Just using the experimental data suggests that $\beta_{\text {eff }} \approx 0.5-0.6$ for $230-300 \mathrm{~K}$, and $\beta_{\text {eff }} \approx 0.4$ at $190 \mathrm{~K}$, giving a superficial picture of the complex growth behavior.

Next, we describe the observed lateral mound coarsening behavior up to $\sim 100 \mathrm{ML}$. The effective coarsening exponent is defined as $n_{\text {eff }}=d(\ln L) / d(\ln \theta)$, where $L$ measures lateral size (see above). Limited statistics preclude precise analysis. However, experimental values for $L_{D}$ and $L_{S}$ in the range of
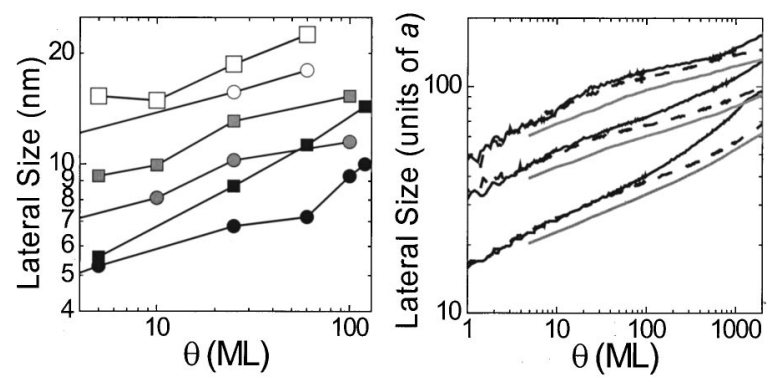

FIG. 2. Lateral mound size $L$ vs coverage $\theta$ for growth at 190 , 230, $260 \mathrm{~K}$ (bottom to top). (a) Experimental: $L_{D}(\bigcirc)<L_{S}(\square)$; measured island densities at $0.1 \mathrm{ML}$ are used to estimate $L_{D}$ for lower $\theta$. (b) Model predictions: $L_{S}$ (gray curves), and $L_{D}$ along close-packed (black dashed) and open (black solid) step directions; $a=2.89 \AA$ is the surface lattice constant.

$5 \mathrm{ML}$ to $60-120 \mathrm{ML}$ [Fig. 2(a)] indicate that $n_{\text {eff }}$ varies much less with $\theta$ than does $\beta_{\text {eff }}$, and generally give values for $n_{\text {eff }}$ consistent with each other, and with simulation predictions [Fig. 2(b)]. We find that $n_{\text {eff }} \approx 0.18(0.19)$ for $260 \mathrm{~K}, \approx 0.18$ $(0.17)$ for $230 \mathrm{~K}$, and a somewhat higher $n_{\text {eff }} \approx 0.2-0.3(0.19)$ at $190 \mathrm{~K}$, from experiment (simulation). Thus, a basic experimental observation is that coarsening is much slower than kinetic roughening up to $\sim 100 \mathrm{ML}$.

Finally, we briefly describe our LEED results for coarsening in the initial stage of growth. Based on behavior of $L_{C}$, we find that $n_{\text {eff }} \approx 0.20$ (up to $\sim 10 \mathrm{ML}$ ) at $190 \mathrm{~K}, n_{\text {eff }}$ $\approx 0.19$ (up to $\sim 15 \mathrm{ML}$ ) at $230 \mathrm{~K}$, and $n_{\text {eff }} \approx 0.3$ (up to $\sim 7$ ML) at $260 \mathrm{~K}$. This increase in the initial $n_{\text {eff }}$ with $T$ is compatible with simulation results up to $\sim 10 \mathrm{ML}$ (where $n_{\mathrm{eff}} \approx 0.20$ at 190 and $230 \mathrm{~K}$, and $n_{\mathrm{eff}} \approx 0.22$ at $260 \mathrm{~K}$ ), but differs from behavior for the subsequent preasymptotic regime.

There are some limitations in our analysis. The STM tip cannot fully probe the floor of narrow and deep valleys (see the Zeno effect ${ }^{16}$ ) resulting in a potential underestimation of $W$, and an excessively positive skewness of the film height distribution. ${ }^{17}$ This effect is likely significant at $230 \mathrm{~K}$ where mounds are fairly small and growth from $25-100 \mathrm{ML}$ is roughest. Indeed, the experimental $W=1.9$ at $100 \mathrm{ML}$ (not shown in Fig. 1) is well below the simulated value. Also, ambiguities in "mound" identification at $190 \mathrm{~K}$ (and at all $T$ for low $\theta$ ) make analysis of $L$ difficult, and simulated $L$ values somewhat exceed experiment for 230-260 K.

Next, we describe our atomistic modeling of the growth process. Our philosophy is to tailor our model to the Ag/ $\operatorname{Ag}(100)$ system, emphasizing a few key atomistic processes whose barriers are free parameters. In this way, the key processes are clarified, and their barriers determined by matching experiment. Specifically, we incorporate the following steps: deposition of atoms randomly at fourfold hollow sites of a fcc(100) surface according to downward funneling deposition dynamics, ${ }^{14}$ adatoms then diffuse across the film surface irreversibly nucleating new islands when two meet, and irreversibly aggregating with existing islands upon reaching their edges. Intralayer terrace diffusion of isolated adatoms occurs with attempt frequency $\nu=10^{13} \mathrm{~s}^{-1}$ and activation barrier $E_{d}=0.40 \mathrm{eV}$; we include an additional SE barrier of 


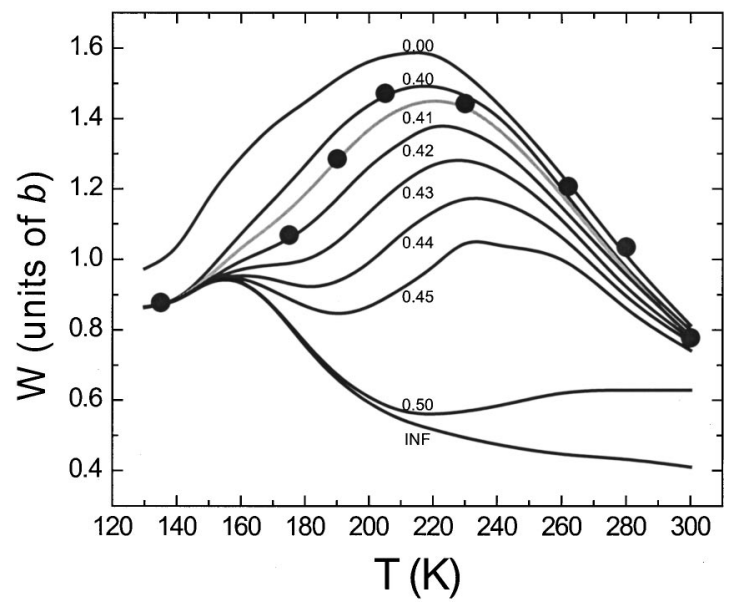

FIG. 3. $W$ vs $T$ for 25 ML films predicted by the atomistic model for various kink rounding barriers $E_{\mathrm{KR}}$ (shown). EKR corresponds to $E_{\mathrm{KR}}=0$. Experimental values are shown as symbols.

$E_{\mathrm{SE}}$ for downward hops at close-packed [110] step edges, but no SE barrier at open or kinked step edges. This choice of nonuniform SE barrier ${ }^{18}$ is motivated by semiempirical studies of energetics. ${ }^{19}$ Finally, we must prescribe the periphery diffusion of adatoms at island edges. In our simplest model, ${ }^{17}$ such adatoms move immediately to double-bonded kink sites, even if this involves kink rounding. This "efficient kink rounding" (EKR) model reasonably describes growth coalescence of islands in the same layer, which is key to predicting $L_{D}$. In fact, choosing $E_{\mathrm{SE}}=0.07 \mathrm{eV}$, it recovers the observed $W$ and $L_{D}$ in $25 \mathrm{ML}$ films for all $T \geqslant 230 \mathrm{~K}$ (see Fig. 3).

The EKR model produces near-square islands whose [110] edges have a "high" ES barrier. However, kink rounding is likely inhibited at lower $T$, producing irregular island shapes with a higher population of kinked step edges having no ES barrier. This explains why the EKR model overestimates $W$ for 25 ML films deposited at lower $T$. Thus, we refine our model to incorporate "restricted kink rounding" (RKR) controlled by a barrier $E_{\mathrm{KR}}$ (which must exceed the low barrier of $0.25 \mathrm{eV}$ for hopping along perfect [110] step edges ${ }^{15}$ ). Figure 3 shows that $W$ for $25 \mathrm{ML}$ films at low $T$ depends very sensitively on $E_{\mathrm{KR}}$, which is selected as 0.41 $\mathrm{eV}$ (for $\nu_{\text {edge }}=10^{12} \mathrm{~s}^{-1}$ ) to match the observed behavior. To summarize, reentrant smooth growth of $\sim 25 \mathrm{ML}$ films at low $T$ is due in part to enhanced downward funneling (note EKR model results), and in part to the development of irregular islands (resulting from RKR) with no ES barrier along kinked step edges.

Our model successfully reproduces the key features of roughening and mound coarsening observed for growth up to 60-100 ML (see Figs. 1, 2, 4). Thus, we believe it reliably predicts growth for thick films, including the extended mound steepening regime, and the transition to and evolution in the slope selection regime. The following discussion focuses on these regimes, comparing predicted behavior with various existing concepts and theories for mound coarsening.

Preasymptotic mound steepening regime. Perhaps the key observation here is that the smaller island separations, and
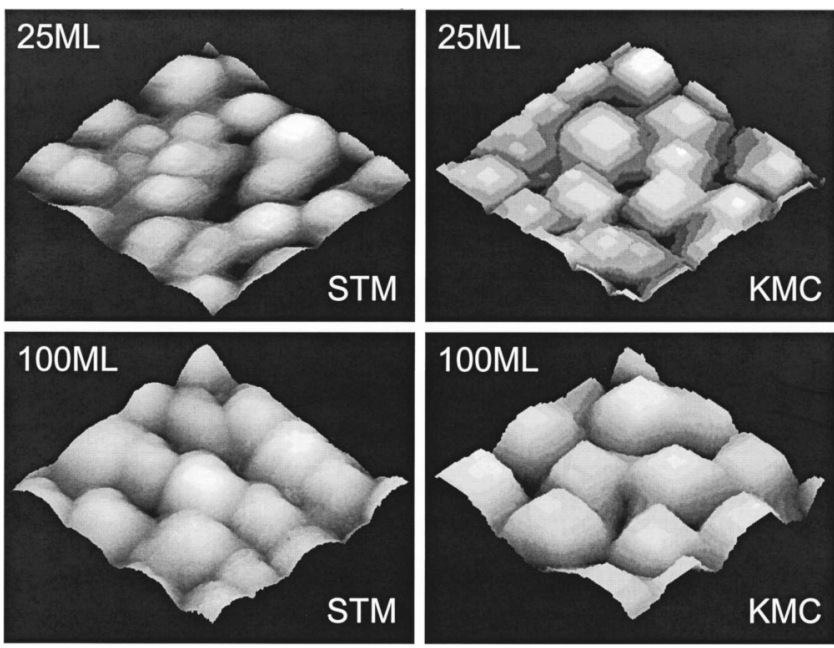

FIG. 4. Film morphologies $\left(50 \times 50 \mathrm{~nm}^{2}\right)$ at $230 \mathrm{~K}$ from: (a) STM experiment; (b) simulations.

thus smaller lateral mound sizes $L_{D}$ at low $T$ result in a more rapid increase in slope, and thus earlier slope selection. ${ }^{20}$ Unexpected rough growth at $300 \mathrm{~K}$ is a consequence of the feature that large island separations (and thus $L_{D}$ ) allow prolonged steepening. It is instructive to compare observed behavior with Golubovic's predictions of rapid roughening with $\beta=\frac{1}{2}$, and slow coarsening with $n_{\text {eff }}=\frac{1}{6}$ from simple continuum models for growth without slope selection, ${ }^{10}$ and with a "relaxation term" tailored to irreversible island formation. ${ }^{7}$ While it is too simplistic to say $\beta=\frac{1}{2}$ for this system, this analysis does seem to capture the basic behavior in the preasymptotic regime. A more general perspective motivated by previous simulations, ${ }^{21}$ which seems to apply here, is that coarsening may be slow when steepening is operative, due to competition between these processes. Finally, it is appropriate to note that the possibility of rapid roughening following initial smooth growth for $\mathrm{Ag} / \mathrm{Ag}(100)$ at $300 \mathrm{~K}$ was suggested by previous x-ray scattering studies, ${ }^{4}$ but it was not pursued or explained.

Asymptotic slope-selection regime. Values of exponents $\beta \approx n_{\text {eff }}$ from $0.25-0.3$ are also consistent with standard continuum models ${ }^{22}$ for growth. ${ }^{11,12}$ The trend of (slightly) higher $n_{\text {eff }}$ for lower $T$ differs from previous simulations where inhibited kink rounding produced lower $n_{\text {eff }}{ }^{12}$ suggesting that the nonuniform SE barrier in our system likely

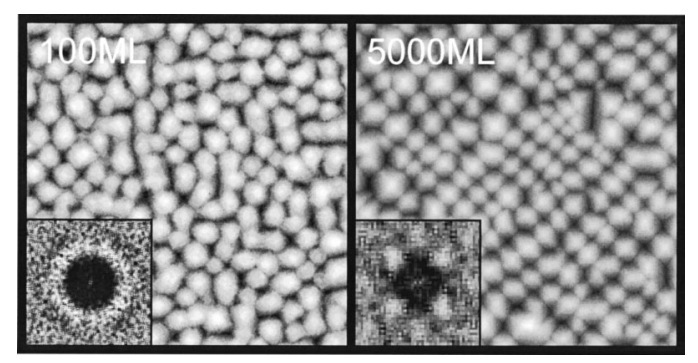

FIG. 5. Mound ordering at $190 \mathrm{~K}$ in our model for $100 \mathrm{ML}$ $\left(130 \times 130 \mathrm{~nm}^{2}\right)$ and $5000 \mathrm{ML}\left(300 \times 300 \mathrm{~nm}^{2}\right)$ films; [100] steps are horizontal. Insets: power spectrum for mound centers. 


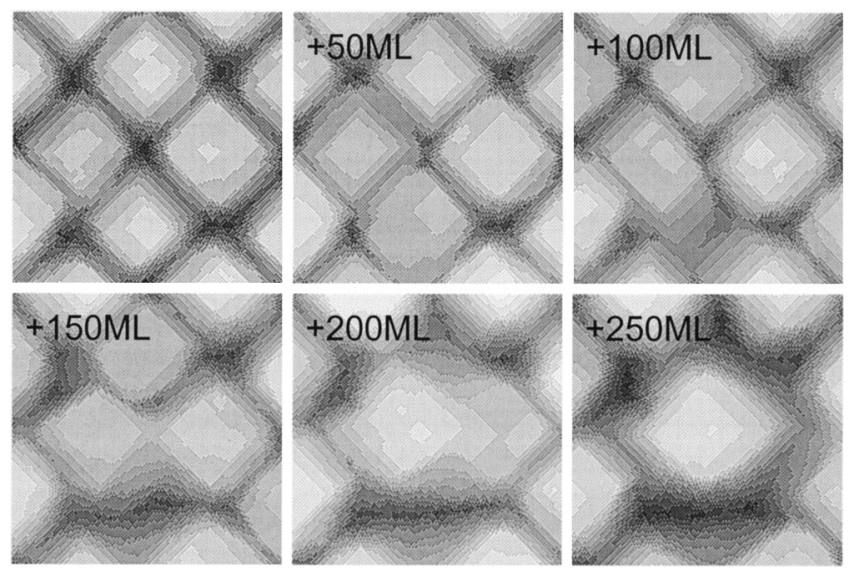

FIG. 6. Complex mound dynamics within ordered $1 \times 1$ patches at $230 \mathrm{~K}$ in the atomistic model. Images are $35 \times 35 \mathrm{~nm}^{2}$. Coverage increments are $50 \mathrm{ML}$.

produces modified behavior. However, a major difference in the dynamics of mounds emerges between our model and previous continuum models incorporating up-down symmetry. In the latter, a checkerboard pattern of alternating mounds and pits (inverted mounds) develops, and evolution is enslaved to the dynamics of rooftop or valley floor "defects." 11

Our model for $\mathrm{Ag} / \mathrm{Ag}(100)$ growth reveals a roughly isotropic distribution of mounds at the beginning of the asymptotic regime, from which develops ordered $1 \times 1$ patches of mounds with square bases along the close-packed directions (Fig. 5). Correspondingly, the power spectrum of the distribution of mound centers evolves from an isotropic ring to a fourfold symmetric pattern (Fig. 5), and $L_{D}$ in [110] and [100] step directions start equal, but ultimately differ by $\sim \sqrt{2}$ [Fig. 2(b)]. This reflects strong up-down symmetry breaking, with valley floors (which separate most mounds) greatly favored over rooftops. Mound dynamics differs qualitatively from Ref. 11. For disordered arrays of square mounds, corner-to-corner coalescence seems to predominate. However, ordered $1 \times 1$ regions of side-by-side mounds display a more complex and cooperative dynamics: fluctuations in size of adjacent mounds trigger annihilation of the smaller neighbor, leading to corner-to-corner coalescence (and other synchronous annihilation) events, see Fig. 6. Corner-tocorner coalescence requires considerable disruption of the ordered $1 \times 1$ pattern.

In summary, we have presented a detailed picture of growth in $\mathrm{Ag} / \mathrm{Ag}(100)$ homoepitaxy, revealing unexpected rough growth at $300 \mathrm{~K}$ due to prolonged mound steepening, and providing a picture of the highly cooperative long-time dynamics of mounds with selected slope.

This work was supported by NSF Grant No. CHE0078596 at Ames Laboratory-U.S. DOE, which is operated by ISU under Contract No. W-7405-Eng-82. J.W.E. acknowledges a useful discussion with Thomas Michely.
${ }^{1}$ A. J. Bray, Adv. Phys. 43, 357 (1994).

${ }^{2}$ M. D. Johnson et al., Phys. Rev. Lett. 72, 116 (1994).

${ }^{3}$ J. Vrijmoeth et al., Phys. Rev. Lett. 72, 3843 (1994).

${ }^{4}$ W. C. Elliott et al., Phys. Rev. B 54, 17938 (1996).

${ }^{5}$ M. Kalff et al., Surf. Sci. Lett. 426, L447 (1999).

${ }^{6}$ H. J. Ernst et al., Phys. Rev. Lett. 72, 112 (1994).

${ }^{7}$ J. A. Stroscio et al., Phys. Rev. Lett. 75, 4246 (1995).

${ }^{8}$ C. R. Stoldt et al., Phys. Rev. Lett. 85, 800 (2000).

${ }^{9}$ G. Constantini et al., Phys. Rev. Lett. 86, 838 (2001).

${ }^{10}$ L. Golubovic, Phys. Rev. Lett. 78, 90 (1997).

${ }^{11}$ M. Siegert, Phys. Rev. Lett. 81, 5481 (1998); D. Moldovan and L. Golubovic, Phys. Rev. E 61, 6190 (2000).

${ }^{12}$ L. Tang et al., Eur. Phys. J. B 2, 409 (1998); S. Schinzer et al., Surf. Sci. 439, 191 (1999); J. G. Amar, Phys. Rev. B 60, R11 317 (1999); T. Michely et al., Phys. Rev. Lett. 86, 2589 (2001) suggest that coarsening requires escape of atoms from kinks. This applies only during mound steepening for systems with large $E_{\mathrm{SE}}$ (the latter condition is not satisfied here).

${ }^{13}$ J. Villain, J. Phys. I 1, 19 (1991).
${ }^{14}$ M. C. Bartelt and J. W. Evans, Phys. Rev. Lett. 75, 4250 (1995); Surf. Sci. 423, 189 (1999).

${ }^{15}$ B. D. Yu and M. Scheffler, Phys. Rev. Lett. 77, 1095 (1996).

${ }^{16}$ P. Politi and J. Villain, Phys. Rev. B 54, 5114 (1996).

${ }^{17}$ K. J. Caspersen et al., Phys. Rev. B 63, 085401 (2001); K. J. Caspersen and J. W. Evans, in Proceedingings of NATO ARW on Atomistic Aspects of Epitaxial Growth (Kluwer, Dordrecht, 2002).

${ }^{18}$ Our modeling with a uniform ES barrier fails to describe observed roughening.

${ }^{19}$ U. Kurpick and T. S. Rahman, Phys. Rev. B 57, 2482 (1998).

${ }^{20}$ This applies even though selected slopes are somewhat higher at lower $T$ (Ref. 17).

${ }^{21}$ P. Smilauer and D. D. Vvedensky, Phys. Rev. B 52, 14263 (1995).

${ }^{22}$ Here the relaxation term has the traditional Mullins form; this term is present and eventually dominates even in systems with irreversible island formation (Ref. 16). 